International Mathematical Forum, Vol. 9, 2014, no. 21, 1029 - 1033

HIKARI Ltd, www.m-hikari.com

http://dx.doi.org/10.12988/imf.2014.4596

\title{
Algebras of Distributions of Binary Semi-isolating Formulas for Families of Isolated Types and for Countably Categorical Theories
}

\author{
Sergey V. Sudoplatov \\ Sobolev Institute of Mathematics \\ Academician Koptyug avenue, 4 \\ 630090, Novosibirsk, Russia \\ $\&$ \\ Novosibirsk State Technical University \\ K. Marx avenue, 20, 630073, Novosibirsk, Russia \\ $\&$ \\ Novosibirsk State University \\ Pirogova street, 2, 630090, Novosibirsk, Russia \\ Copyright (C) 2014 Sergey V. Sudoplatov. This is an open access article distributed under \\ the Creative Commons Attribution License, which permits unrestricted use, distribution, \\ and reproduction in any medium, provided the original work is properly cited.
}

\begin{abstract}
We apply a general approach for distributions of binary isolating and semi-isolating formulas to families of isolated types and to the class of countably categorical theories. Containing compositions and Boolean combinations, structures associated with binary formulas linking isolated types in general case and for $\omega$-categorical theories are characterized.
\end{abstract}

Mathematics Subject Classification: 03C07, 03G15, 20N02, 08A02, 08A55

Keywords: structure of binary semi-isolating formulas, isolated type, countably categorical theory 


\section{Introduction}

Algebras and structures associated with isolating and semi-isolating formulas of a theory are introduced in [1,2]. These derived structures allow to find basic structural properties, to describe theories and their models on binary level, as well as to classify them. We apply the general approach for distributions of formulas to families of isolated types and to an important class of elementary theories: the class of $\omega$-categorical theories.

The paper is organized as follows. In Section 2 we recall definitions for structures of distributions of formulas. In Section 3 we prove basic properties for algebras of binary formulas linking isolated types (Proposition 3.1) and, in particular, for algebras associated with $\omega$-categorical theories (Corollary 3.2). It is shown that an abstract algebra with the basic properties can be realized as an algebra of distributions of binary semi-isolating formulas linking isolated types of a theory and, moreover, this theory can be chosen $\omega$-categorical (Theorem 3.3, Corollary 3.4).

\section{Algebras of distributions of binary isolating and semi-isolating formulas}

Definition $2.1[1,2,3,4]$. Let $T$ be a complete theory, $\mathcal{M} \models T$. Consider types $p(x), q(y) \in S(\emptyset)$, realized in $\mathcal{M}$, and all $(p, q)$-preserving, or semiisolating, formulas $\varphi(x, y)$ of $T$, i. e., formulas for which there is $a \in M$ such that $\models p(a)$ and $\varphi(a, y) \vdash q(y)$. Now, for each such a formula $\varphi(x, y)$, we define a binary relation $R_{p, \varphi, q}=\{(a, b) \mid \mathcal{M}=p(a) \wedge \varphi(a, b)\}$. If $(a, b) \in R_{p, \varphi, q},(a, b)$ is called a $(p, \varphi, q)$-arc. If $\varphi(a, y)$ is principal (over $a)$, the $(p, \varphi, q)$-arc $(a, b)$ is also principal.

If $\varphi(x, y)$ is a $(p \leftrightarrow q)$-formula, i. e., it is both a $(p \rightarrow q)$ - and a $(q \rightarrow p)$ formula then the set $[a, b]=\{(a, b),(b, a)\}$ is said to be a $(p, \varphi, q)$-edge. If the $(p, \varphi, q)$-edge $[a, b]$ consists of principal $(p, \varphi, q)$ - and $(q, \varphi(y, x), p)$-arcs then $[a, b]$ is a principal $(p, \varphi, q)$-edge.

$(p, \varphi, q)$-arcs and $(p, \varphi, q)$-edges are called arcs and edges respectively if we say about fixed or some formula $\varphi(x, y)$. If $(a, b)$ is a $(p, \varphi, q)$-arc such that the pair $(b, a)$ is not an arc for any $(q, p)$-formula, then $(a, b)$ is called irreversible.

For types $p(x), q(y) \in S(\emptyset)$, we denote by $\operatorname{PF}(p, q)$ the set

$$
\{\varphi(x, y) \mid \varphi(a, y) \text { is a principal formula, } \varphi(a, y) \vdash q(y) \text {, where } \models p(a)\} \text {. }
$$

Let $\operatorname{PE}(p, q)$ be the set of pairs of formulas $(\varphi(x, y), \psi(x, y)) \in \operatorname{PF}(p, q)$ such that for any (some) realization $a$ of $p$ the sets of solutions for $\varphi(a, y)$ and $\psi(a, y)$ coincide. Clearly, $\operatorname{PE}(p, q)$ is an equivalence relation on the set $\operatorname{PF}(p, q)$. 
Notice that each $\mathrm{PE}(p, q)$-class $E$ corresponds to either a principal edge or to an irreversible principal arc connecting realizations of $p$ and $q$ by some (any) formula in $E$. Thus the quotient $\operatorname{PF}(p, q) / \mathrm{PE}(p, q)$ is represented as a disjoint union of sets $\operatorname{PFS}(p, q)$ and $\operatorname{PFN}(p, q)$, where $\operatorname{PFS}(p, q)$ consists of $\operatorname{PE}(p, q)$ classes corresponding to principal edges and $\operatorname{PFN}(p, q)$ consists of $\operatorname{PE}(p, q)$ classes corresponding to irreversible principal arcs.

Let $T$ be a complete theory without finite models, $U=U^{-} \dot{U}\{0\} \dot{U} U^{+}$be an alphabet of cardinality $\geq|S(T)|$, consisting of negative elements $u^{-} \in U^{-}$, positive elements $u^{+} \in U^{+}$, and zero 0 . As usual, we write $u<0$ for any $u \in U^{-}$and $u>0$ for any $u \in U^{+}$. The set $U^{-} \cup\{0\}$ is denoted by $U^{\leq 0}$ and $U^{+} \cup\{0\}$ is denoted by $U^{\geq 0}$. Elements of $U$ are called labels.

Let $\nu(p, q): \operatorname{PF}(p, q) / \mathrm{PE}(p, q) \rightarrow U$ be an injective labelling functions, $p(x), q(y) \in S(\emptyset)$, for which negative elements correspond to classes in $\operatorname{PFN}(p, q) / \operatorname{PE}(p, q)$ and non-negative elements correspond to classes in $\operatorname{PFS}(p, q) / \operatorname{PE}(p, q)$ such that 0 is defined only for $p=q$ and is represented by the formula $(x \approx y), \nu(p)=\nu(p, p)$. We additionally suppose that $\rho_{\nu(p)} \cap \rho_{\nu(q)}=$ $\{0\}$ for $p \neq q$ (where, as usual, we denote by $\rho_{f}$ the image of the function $f$ ) and $\rho_{\nu(p, q)} \cap \rho_{\nu\left(p^{\prime}, q^{\prime}\right)}=\emptyset$ if $p \neq q$ and $(p, q) \neq\left(p^{\prime}, q^{\prime}\right)$. Labelling functions with the properties above as well families of these functions are said to be regular. Below we shall consider only regular labelling functions and their regular families. We denote by $\theta_{p, u, q}(x, y)$ a formula in $\operatorname{PF}(p, q)$ with the label $u \in \rho_{\nu(p, q)}$. If a type $p$ is fixed and $p=q$ then a formula $\theta_{p, u, q}(x, y)$ is denoted by $\theta_{u}(x, y)$.

For types $p_{1}, p_{2}, \ldots, p_{k+1} \in S^{1}(\emptyset)$ and sets $X_{1}, X_{2}, \ldots, X_{k} \subseteq U$ of labels we denote by $P\left(p_{1}, X_{1}, p_{2}, X_{2}, \ldots, p_{k}, X_{k}, p_{k+1}\right)$ the set of all labels $u \in U$ corresponding to formulas $\theta_{p_{1}, u, p_{k+1}}(x, y)$ satisfying, for realizations $a$ of $p_{1}$ and some $u_{1} \in X_{1}, \ldots, u_{k} \in X_{k}$, the following condition:

$$
\theta_{p_{1}, u, p_{k+1}}(a, y) \vdash \theta_{p_{1}, u_{1}, p_{2}, u_{2}, \ldots, p_{k}, u_{k}, p_{k+1}}(a, y),
$$

where

$$
\begin{gathered}
\theta_{p_{1}, u_{1}, p_{2}, u_{2}, \ldots, p_{k}, u_{k}, p_{k+1}}(x, y)= \\
=\exists x_{2}, x_{3}, \ldots x_{k}\left(\theta_{p_{1}, u_{1}, p_{2}}\left(x, x_{2}\right) \wedge \theta_{p_{2}, u_{2}, p_{3}}\left(x_{2}, x_{3}\right) \wedge \ldots\right. \\
\left.\ldots \wedge \theta_{p_{k-1}, u_{k-1}, p_{k}}\left(x_{k-1}, x_{k}\right) \wedge \theta_{p_{k}, u_{k}, p_{k+1}}\left(x_{k}, y\right)\right) .
\end{gathered}
$$

Thus the Boolean $\mathcal{P}(U)$ of $U$ is the universe of an algebra of distributions of binary isolating formulas with $k$-ary operations $P\left(p_{1}, \cdot, p_{2}, \cdot, \ldots, p_{k}, \cdot, p_{k+1}\right)$, where $p_{1}, \ldots, p_{k+1} \in S^{1}(\emptyset)$. This algebra has a natural restriction to any family $R \subseteq S^{1}(\emptyset)$ forming $I_{\mathcal{R}}$-structure.

If all types $p_{i}$ equal to a type $p$ then we write $P_{p}\left(X_{1}, X_{2}, \ldots, X_{k}\right)$ and $P_{p}\left(u_{1}, u_{2}, \ldots, u_{k}\right)$ as well as $\left\lfloor X_{1}, X_{2}, \ldots, X_{k}\right\rfloor_{p}$ and $\left\lfloor u_{1}, u_{2}, \ldots, u_{k}\right\rfloor_{p}$ instead of $P\left(p_{1}, X_{1}, p_{2}, X_{2}, \ldots, p_{k}, X_{k}, p_{k+1}\right)$ and $P\left(p_{1}, u_{1}, p_{2}, u_{2}, \ldots, p_{k}, u_{k}, p_{k+1}\right)$ respectively. We omit the index ${ }_{p}$ if the type $p$ is fixed and write simply $u_{1} \cdot u_{2}$ or $u_{1} u_{2}$ instead of $\left\lfloor u_{1}, u_{2}\right\rfloor$. 
Extending the set $U$ of labels by positive and negative labels for semiisolating formulas as well as by neutral labels $u^{\prime} \in U^{\prime}$ (collecting irreversible arcs and principal edges in a set of solutions of a semi-isolating formula), similar $\mathrm{SI}_{\mathcal{R}}$-structures $S I$ for semi-isolating formulas as well as si-ranks, Boolean operations for labels of these formulas, relations of domination of labels with respect to $\vdash$, and $\mathrm{POSTC}_{\mathcal{R}}$-structures, containing all attributes above, are defined in [2].

\section{Results}

Proposition 3.1 For any theory $T$, a nonempty family $R \subseteq S^{1}(\emptyset)$ of isolated types, and a regular family $\nu(R)$ of labelling functions for semi-isolating formulas, the POSTC $\mathrm{P}_{\mathcal{R}}$-structure $M_{\nu(R)}$ consists of positive labels and zero, and each label $u$ has a complement $\bar{u}$ such that $u \wedge \bar{u}=\emptyset$ and $u \vee \bar{u}$ is a maximal element. If $R=\{p\}$ then the monoid $\mathrm{SI}_{\nu(p)}=\left\langle M_{\nu(R)}, \cdot\right\rangle$ (for compositions of labels) is generated by a Boolean algebra, for which labels $u \vee \bar{u}$ correspond to isolating formulas of $p$.

Proof. The inclusion $\bigcup_{p, q \in R} \rho_{\nu(p, q)} \subseteq U^{\geq 0}$ is proved in Proposition 1.1 [2]. By the definition, for any pair $(p, q) \in R^{2}$ of types, each label $v_{q}$, corresponding to an isolating formula $\varphi_{q}(y)$ of type $q(y)$, is a maximal element (among labels in $\left.\rho_{\nu(p, q)}\right)$. Then for any label $u \in \rho_{\nu(p, q)}$, the label $\neg u \wedge v_{q}$ is the complement $\bar{u}$. It remains to note that for any isolated type $p(x)$, labels in $\rho_{\nu(p)}$ form a Boolean algebra with the least element $\emptyset$ and the greatest element $v_{p}$ such that for any label $u \in \rho_{\nu(p)}, u \wedge \bar{u}=\emptyset$ and $u \vee \bar{u}=v_{p}$.

Proposition is proved.

By Ryll-Nardzewski theorem and Proposition 3.1, we obtain

Corollary 3.2 For any $\omega$-categorical theory $T$, a nonempty family $R \subseteq$ $S^{1}(\emptyset)$, and a regular family $\nu(R)$ of labelling functions for semi-isolating formulas, the $\mathrm{POSTC}_{\mathcal{R}}$-structure $M_{\nu(R)}$ is finite, consists of positive labels and zero, and each label $u$ has a complement $\bar{u}$.

Theorem 3.3 For any $\mathrm{POSTC}_{\mathcal{R}}$-structure $M$, in which each label is positive or zero and has a complement, there is a theory $T$, a nonempty family $R \subseteq S^{1}(\emptyset)$ of isolated types, and a regular family $\nu(R)$ of labelling functions for semi-isolating formulas such that $M_{\nu(R)}=M$.

Proof. Consider the construction for the proof of Theorem 8.1 [2]. We define a family of isolated 1-types bijective with the set $\mathcal{R}$ by disjoint unary predicates $\operatorname{Col}_{p}, p \in \mathcal{R}$. Here we assume that if $\rho_{\nu(p)}$ consists of one (nonempty) 
label, i. e., $\rho_{\nu(p)}=\{0\}$, then $\left|\operatorname{Col}_{p}\right|=1$, and if $\left|\rho_{\nu(p)}\right|>1$ then $\operatorname{Col}_{p}$ contains infinitely many elements. Besides, we assume that each formula $\mathrm{Col}_{p}(x)$ isolates a type marked by $p$. Further scheme is based on a generic construction coordinated with operations in the $\mathrm{POSTC}_{\mathcal{R}}$-structure $M$ and with an ordering (isomorphic to the ordering of labels in $M$ ) of formulas $\theta_{p, u, q}(x, y)$, witnessing that realizations of $p$ semi-isolate realizations of $q, u \in \rho_{\nu(p, q)}, p, q \in \mathcal{R}$, and forming with predicates $\mathrm{Col}_{p}$ the language of theory under construction. Since the formulas $\operatorname{Col}_{q}(y), q \in \mathcal{R}$, are isolated, then for any label $u \in \rho_{\nu(p, q)}$ and any realization $a$ of $p$, we can define formulas $\theta_{p, u, q}(a, y)$ and $\theta_{p, \bar{u}, q}(a, y)$ so that these formulas complement each other in $\operatorname{Col}_{q}(y): \theta_{p, u, q}(a, y) \wedge \theta_{p, \bar{u}, q}(a, y) \vdash$ and $\theta_{p, u, q}(a, y) \vee \theta_{p, \bar{u}, q}(a, y) \equiv \operatorname{Col}_{q}(y)$. The generic construction allows to get a required theory with quantifier elimination.

Having a finite POSTC $_{\mathcal{R}}$-structure $M$ the schema above, generating a theory with quantifier elimination, produce a required theory being $\omega$-categorical:

Corollary 3.4 For any finite POSTC $_{\mathcal{R}}$-structure $M$, in which each label is positive or zero and has a complement, there is an $\omega$-categorical theory $T$, a nonempty family $R \subseteq S^{1}(\emptyset)$, and a regular family $\nu(R)$ of labelling functions for semi-isolating formulas such that $M_{\nu(R)}=M$.

Acknowledgements. The research was supported by RFBR (grant 1201-00460-a).

\section{References}

[1] I.V. Shulepov, S.V. Sudoplatov, Algebras of distributions for isolating formulas of a complete theory, Siberian Electronic Mathematical Reports, 11 (2014), 380 - 407.

[2] S.V. Sudoplatov, Algebras of distributions for semi-isolating formulas of a complete theory, Siberian Electronic Mathematical Reports, 11 (2014), $408-433$.

[3] S.V. Sudoplatov, The Lachlan problem, Edition of Novosibirsk State Technical University, Novosibirsk, 2009. [in Russian; English version is available in: http://www.math.nsc.ru/ sudoplatov/lachlan_eng_03_ 09_2008.pdf]

[4] B.S. Baizhanov, S.V. Sudoplatov, V.V. Verbovskiy, Conditions for nonsymmetric relations of semi-isolation, Siberian Electronic Mathematical Reports, 9 (2012), 161 - 184.

Received: May 11, 2014 